\title{
Neutrino Lines from Majoron Dark Matter
}

\author{
Julian Heeck* \\ Service de Physique Théorique, Université Libre de Bruxelles, CP225, 1050 Brussels, Belgium \\ E-mail: julian.heeck@ulb.ac.be
}

\begin{abstract}
Majorons are the Goldstone bosons associated to lepton number and thus closely connected to Majorana neutrino masses. At tree level, its only fermion couplings are to neutrinos. Couplings to charged fermions arise at one-loop level, including lepton-flavor-violating ones that lead to decays $\ell \rightarrow \ell^{\prime} J$, whereas a coupling to photons is generated at two loops. The typically small couplings make massive majorons a prime candidate for long-lived dark matter. Its signature decay into two mono-energetic neutrinos is potentially detectable for majoron masses above MeV and most importantly depends on different parameters than the visible decay channels.
\end{abstract}

The 19th International Workshop on Neutrinos from Accelerators-NUFACT2017

25-30 September, 2017

Uppsala University, Uppsala, Sweden

${ }^{*}$ Speaker. 


\section{Majoron couplings}

We start our discussion with a basic introduction to the majoron model, based on Refs. [1, 2], before delving into the phenomenology of majorons as dark matter (Sec. 2) and the connection to lepton flavor violation (Sec. 3)

The difference between baryon number $B$ and lepton number $L$ is an anomaly-free global symmetry of the Standard Model (SM); spontaneously breaking this $U(1)_{B-L}$ symmetry results in a Goldstone boson called majoron [3,4]. In the simplest realization, this majoron $J$ resides in a singlet complex scalar $\sigma=\left(f+\sigma^{0}+i J\right) / \sqrt{2}$ that carries $B-L$ charge $2, f$ being the $B-L$ breaking scale and $\sigma^{0}$ the heavy CP-even majoron partner. Further introducing three right-handed neutrinos $N_{R}$, the Lagrangian reads

$$
\mathscr{L}=\mathscr{L}_{\mathrm{SM}}+i \bar{N}_{R} \gamma^{\mu} \partial_{\mu} N_{R}+\left(\partial_{\mu} \sigma\right)^{\dagger}\left(\partial^{\mu} \sigma\right)-V(\sigma)-\left(\bar{L} y N_{R} H+\frac{1}{2} \bar{N}_{R}^{c} \lambda N_{R} \sigma+\text { h.c. }\right),
$$

with the SM lepton (scalar) doublet $L(H)$. We suppressed flavor indices and details of the scalar potential $V(\sigma)$. SU $(2)_{L} \times U(1)_{Y} \times U(1)_{B-L}$ symmetry breaking then yields the famous seesaw neutrino mass matrix $M_{v} \simeq-m_{D} M_{R}^{-1} m_{D}^{T}$ with $m_{D}=y v / \sqrt{2}$ and $M_{R}=\lambda f / \sqrt{2} \gg m_{D}$.

Five of the nine parameters encoded in $M_{v}$ have been measured already: the two mass splittings and three mixing angles. However, even if we could measure all elements of $M_{v}$, we would still not be able to reconstruct the underlying seesaw parameters $m_{D}$ and $M_{R}$. As shown in Ref. [5], one can map the parameters $\left\{m_{D}, M_{R}\right\}$ bijectively onto $\left\{M_{v}, m_{D} m_{D}^{\dagger}\right\}$, implying that $m_{D} m_{D}^{\dagger}$ contains precisely those nine seesaw parameters that cannot be determined by measurements of neutrino masses and oscillations. As we will see below, this is a convenient parametrization for the phenomenology of majorons, which endow $m_{D} m_{D}^{\dagger}$ with physical meaning.

The tree-level couplings of the majoron $J$ can easily be derived from Eq. (1.1), which in particular include the couplings $J \bar{v}_{j} i \gamma_{5} v_{j} m_{j} /(2 f)$ to the light neutrino mass eigenstates $v_{j}$. With $f$ at the seesaw scale and active neutrino masses $m_{j}$ below $\mathrm{eV}$, this coupling is incredibly tiny. At one-loop level $[3,6,1]$, the majoron also obtains couplings to charged leptons $\ell$ and quarks $q$, parametrized as $i J \bar{f}_{1}\left(g_{J f_{1} f_{2}}^{S}+g_{J f_{1} f_{2}}^{P} \gamma_{5}\right) f_{2}$ with coefficients

$$
\begin{array}{llrl}
g_{J q q^{\prime}}^{P} & \simeq \frac{m_{q}}{8 \pi^{2} v} \delta_{q q^{\prime}} T_{3}^{q} \operatorname{tr} K, & g_{J q q^{\prime}}^{S} & =0, \\
g_{J \ell \ell^{\prime}}^{P} & \simeq \frac{m_{\ell}+m_{\ell^{\prime}}}{16 \pi^{2} v}\left(\delta_{\ell \ell^{\prime}} T_{3}^{\ell} \operatorname{tr} K+K_{\ell \ell^{\prime}}\right), & g_{J \ell \ell^{\prime}}^{S} & \simeq \frac{m_{\ell^{\prime}}-m_{\ell}}{16 \pi^{2} v} K_{\ell \ell^{\prime}},
\end{array}
$$

where $T_{3}^{d, \ell}=-T_{3}^{u}=-1 / 2$ and we introduced the dimensionless hermitian coupling matrix $K \equiv$ $m_{D} m_{D}^{\dagger} /(v f)$. The majoron couplings to charged fermions are hence determined by the seesaw parameters $m_{D} m_{D}^{\dagger}$, which are independent of the neutrino masses and can in particular be much bigger than the naive one-generation expectation $M_{v} M_{R}$. Perturbativity sets an upper bound on $K$ of order $4 \pi v / f$, and since $K$ is furthermore positive definite we have the inequalities $\left|K_{\ell \ell^{\prime}}\right| \leq$ $\sqrt{K_{\ell \ell} K_{\ell^{\prime} \ell^{\prime}}} \leq \operatorname{tr} K$. These fermion couplings are obviously crucial for majoron phenomenology and in principle even offer a new avenue to reconstruct the seesaw parameters. Note in particular the off-diagonal lepton couplings, which will lead to lepton flavor violation [6, 1] (Sec. 3).

There is one more coupling of interest, that to photons. For a massless majoron, the coupling $J F \tilde{F}$ vanishes because $B-L$ is anomaly free [6]; otherwise, it is induced at two-loop level and 


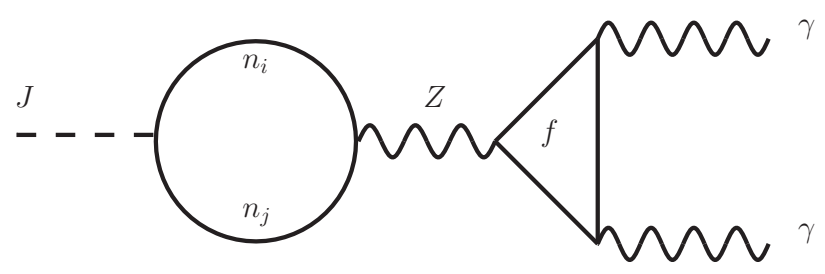

Figure 1: Majoron decay into two photons as mediated by loops of neutrinos $n_{j}$ and charged SM fermions $f$, effectively mixing $J$ with the longitudinal component of $Z$ [1].

non-trivial to calculate. Considering only the gauge-invariant subset of diagrams shown in Fig. 1, we can however obtain the simple expression [1]

$$
\Gamma(J \rightarrow \gamma \gamma) \simeq \frac{\alpha^{2}(\operatorname{tr} K)^{2}}{4096 \pi^{7}} \frac{m_{J}^{3}}{v^{2}}\left|\sum_{f} N_{c}^{f} T_{3}^{f} Q_{f}^{2} g\left(\frac{m_{J}^{2}}{4 m_{f}^{2}}\right)\right|^{2},
$$

where the sum is over all SM fermions $f$ with color multiplicity $N_{c}^{f}$, isospin $T_{3}^{f}$, and electric charge $Q_{f}$. The loop function $g$ is given by

$$
g(x)=-\frac{(\log [1-2 x+2 \sqrt{x(x-1)}])^{2}}{4 x}= \begin{cases}\frac{1}{x}(\arcsin \sqrt{x})^{2} & \text { for } x \leq 1 \\ -\frac{1}{4 x}\left(\log \left[\frac{1+\sqrt{1-\frac{1}{x}}}{1-\sqrt{1-\frac{1}{x}}}\right]-i \pi\right)^{2} & \text { for } x>1\end{cases}
$$

The other two-loop diagrams are more complicated to calculate, but importantly depend on different parameters (and in particular not on quark masses) and thus cannot fully cancel the amplitude. Eq. (1.4) is therefore a reasonable guess for the decay rate.

\section{Majoron dark matter}

With the relevant majoron couplings at our disposal, we can start to discuss phenomenology. First off, we are going to study the case of the majoron as a dark matter (DM) candidate. This is motivated by the fact that it generically has tiny couplings to the SM, ensuring that it is dark and stable enough to form DM [7,8]. A prerequisite here is an explicit $U(1)_{B-L}$ breaking in the Lagrangian to generate a majoron mass $m_{J}$, making $J$ a pseudo-Goldstone boson. This could simply be an explicit mass term in the scalar potential, a gravity-generated higher-dimensional operator or an axion-like anomaly-induced potential. Furthermore, a production mechanism is required to generate the observed abundance in the early Universe. With small couplings, the obvious mechanism to use here is freeze-in, e.g. from the coupling to the Higgs or the right-handed neutrinos [9]. For majoron masses as low as $\mathrm{keV}$ one has to be careful not to violate structureformation constraints from the Lyman- $\alpha$ forest. In these cases, different production mechanisms are required that make $J$ cold enough, which can naturally be found in inverse-seesaw majoron models $[10,11]$. Here we will focus on DM masses above $\mathrm{MeV}$ for simplicity.

Assuming a massive singlet majoron to make up all of DM, the main signature then comes from its eventual decay into SM particles. As discussed above, the only decay channel at tree level is into neutrino mass eigenstates, $J \rightarrow v_{j} v_{j}$, with coupling $m_{j} / f$. These neutrinos will not 

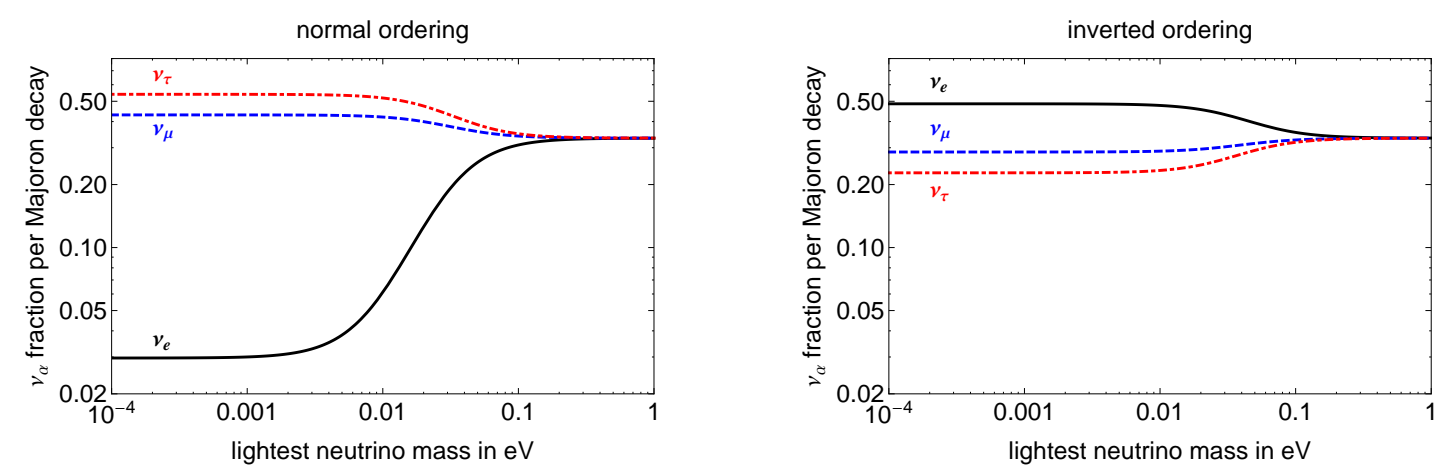

Figure 2: Branching ratios of $J$ decay into the different neutrino flavors $v_{\alpha}$.

oscillate, so the flavor content of the monochromatic neutrino flux follows simply from the mass eigenstates [1]. For normal hierarchy, this implies only a small $v_{e}$ component of the flux, because the heaviest neutrino only has a tiny $\theta_{13}$-suppressed electron component; for inverted hierarchy, the majoron decays into the two heaviest neutrinos, which results in roughly $50 \%$ electron flavor in the flux; in the quasi-degenerate regime, all flavors are equally probable. See Fig. 2 for an illustration.

Knowing the flavor composition of $J \rightarrow v v$ allows us to search for these neutrino lines with neutrino detectors. Borexino and KamLAND use inverse beta decay $\bar{v}_{e} p \rightarrow n e^{+}$to reconstruct the neutrino energy with good accuracy. Due to the kinematic threshold of this process it is not possible to detect neutrino lines below $m_{J} \sim \mathrm{MeV}$. Above $\mathrm{MeV}$, on the other hand, these experiments could indeed be sensitive to a dark-matter induced neutrino flux [1] (see Fig. 3). For higher masses, Super-K becomes most sensitive and can also utilize the $v_{\mu}$ component of the flux [12]. For sub$\mathrm{MeV}$ masses, limits on $J \rightarrow v v$ can still be derived from cosmology [13], but are of course less of a smoking-gun signature for majoron DM.

Majoron DM can thus be used to motivate neutrino line searches all the way down to $\mathrm{MeV}$ energies, far below what is typically considered. A natural question to ask here is whether observable neutrino fluxes are compatible with limits from visible DM decay channels, which are far more constrained. As shown above, the decays $J \rightarrow \ell \overline{\ell^{\prime}}, q \bar{q}, \gamma \gamma$ are indeed all unavoidably induced at loop level in the singlet majoron model. However, they all depend on parameters that are independent of the $J \rightarrow v v$ channel, making it impossible to directly compare these channels. In other words, the DM decay into visible channels probes different parameters than $J \rightarrow v v$, making them complementary. In the $m_{J}=\mathrm{MeV}-100 \mathrm{GeV}$ region, one can indeed obtain strong constraints on the $K$ matrix elements from the visible channels, without invalidating our conclusion about neutrino lines [1]. For sub-MeV majoron masses, only the decay $J \rightarrow \gamma \gamma$ remains as a promising indirect detection signature $[8,14]$.

\section{Lepton flavor violation}

Going back to the majoron couplings to fermions of Eq. (1.3) shows that the quark couplings are diagonal at one-loop level, whereas the lepton couplings are not. Due to the rather strong lepton mass hierarchy, $m_{\ell} \gg m_{\ell^{\prime}}$, the off-diagonal couplings can be approximately writ- 


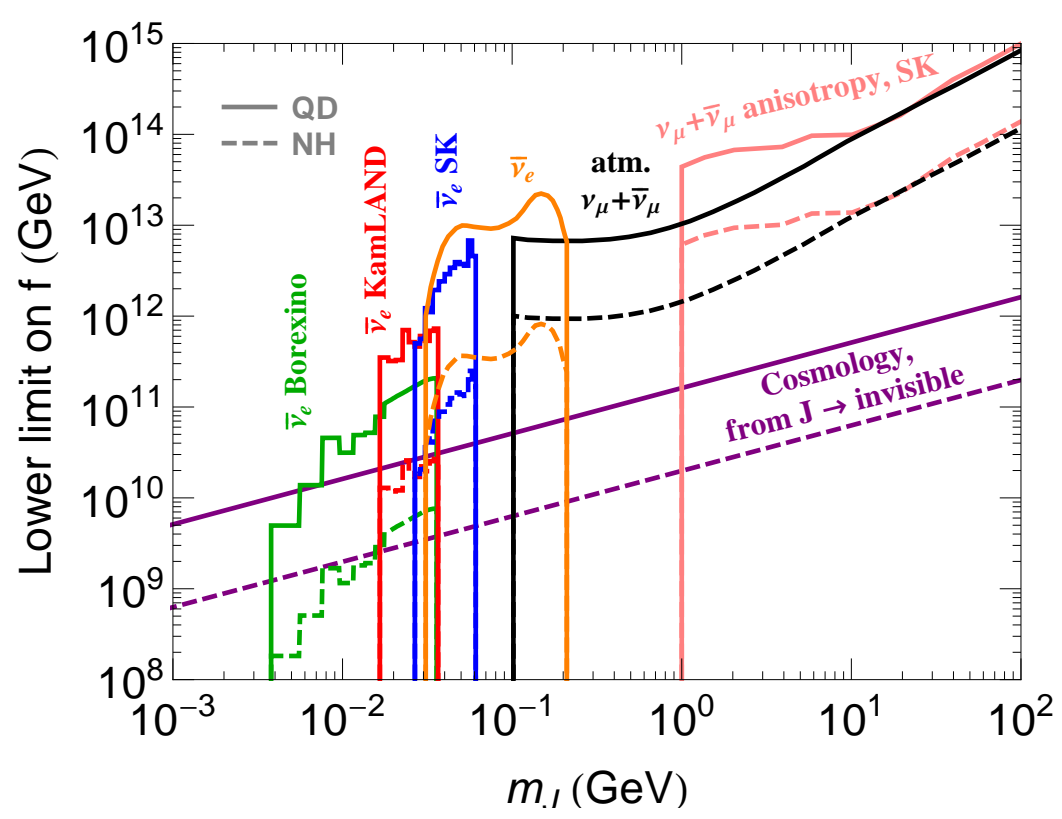

Figure 3: Lower limit on the $B-L$ breaking scale $f$ from DM decay $J \rightarrow v v$, assuming a quasi-degenerate (solid) or normal-hierarchy neutrino spectrum (dashed) [1].

ten as $-\frac{i m_{\ell}}{8 \pi^{2} v} K_{\ell \ell^{\prime}} J \bar{\ell} P_{L} \ell^{\prime}+$ h.c., which can induce the lepton-flavor-violating two-body decays $\ell \rightarrow$ $\ell^{\prime} J[6,1]$. If the majoron is massless or decays invisibly, the only signature of this decay is the mono-energetic $\ell^{\prime}$, which has to be searched for on top of the continuous energy spectrum from the SM decay channel $\ell \rightarrow \ell^{\prime} v_{\ell} \bar{v}_{\ell^{\prime}}$. Current limits translate into $\left|K_{\mu e}\right| \lesssim 10^{-5},\left|K_{\tau \ell}\right| \lesssim \mathscr{O}\left(10^{-3}\right)$, with good prospects for improvement at Mu3e and Belle $[15,16]$. Channels with more tagging potential, such as $\ell \rightarrow \ell^{\prime} J \gamma$ or $\ell \rightarrow \ell^{\prime}(J \rightarrow$ visible $)$, are also promising and can severely improve sensitivity [17]. We stress that lepton flavor violation with majorons depends on a different combination of seesaw parameters than the more commonly studied heavy-neutrino induced $\ell \rightarrow \ell^{\prime} \gamma$. These channels are therefore complementary and should both be investigated.

\section{Conclusion}

The singlet majoron model inherits some nice properties from the seesaw Lagrangian, namely small Majorana neutrino masses and leptogenesis, while providing a new phenomenological handle. The loop-induced majoron couplings to charged particles are precisely given by the seesaw parameters that are impossible to determine from the neutrino mass matrix, which could in principle allow us to reconstruct the seesaw with low-energy measurements. Since the couplings can be tiny without fine-tuning, a massive majoron makes for a promising unstable dark matter candidate, with signature decay into mono-energetic neutrinos, potentially detectable for energies above $\mathrm{MeV}$. With few new parameters, which are furthermore linked to the seesaw mechanism, majoron models are simple extensions of the Standard Model that still provide rich phenomenology. 


\section{Acknowledgments}

I thank the NUFACT organizers for inviting me to this interesting and stimulating conference, as well as Camilo Garcia-Cely for collaboration on the work presented here. This work was supported by the F.R.S.-FNRS.

\section{References}

[1] C. Garcia-Cely and J. Heeck, Neutrino Lines from Majoron Dark Matter, JHEP 05 (2017) 102, [1701.07209].

[2] J. Heeck, Phenomenology of Majorons, in 13th Patras Workshop on Axions, WIMPs and WISPs, Thessaloniki, Greece, May 15-19, 2017, 1709.07670.

[3] Y. Chikashige, R. N. Mohapatra and R. D. Peccei, Are There Real Goldstone Bosons Associated with Broken Lepton Number?, Phys. Lett. B98 (1981) 265-268.

[4] J. Schechter and J. W. F. Valle, Neutrino Decay and Spontaneous Violation of Lepton Number, Phys. Rev. D25 (1982) 774.

[5] S. Davidson and A. Ibarra, Determining seesaw parameters from weak scale measurements?, JHEP 09 (2001) 013, [hep-ph/ 0104076$].$

[6] A. Pilaftsis, Astrophysical and terrestrial constraints on singlet Majoron models, Phys. Rev. D49 (1994) 2398-2404, [hep-ph/9308258].

[7] I. Z. Rothstein, K. S. Babu and D. Seckel, Planck scale symmetry breaking and majoron physics, Nucl. Phys. B403 (1993) 725-748, [hep-ph/9301213].

[8] V. Berezinsky and J. W. F. Valle, The KeV majoron as a dark matter particle, Phys. Lett. B318 (1993) 360-366, [hep-ph/9309214].

[9] M. Frigerio, T. Hambye and E. Masso, Sub-GeV dark matter as pseudo-Goldstone from the seesaw scale, Phys. Rev. X1 (2011) 021026, [1107.4564].

[10] J. Heeck and D. Teresi, Cold keV dark matter from decays and scatterings, Phys. Rev. D96 (2017) 035018, [1706.09909].

[11] S. Boulebnane, J. Heeck, A. Nguyen and D. Teresi, Cold light dark matter in extended seesaw models, 1709.07283.

[12] S. Palomares-Ruiz, Model-Independent Bound on the Dark Matter Lifetime, Phys. Lett. $\mathbf{B 6 6 5}$ (2008) 50-53, [0712.1937].

[13] V. Poulin, P. D. Serpico and J. Lesgourgues, A fresh look at linear cosmological constraints on a decaying dark matter component, JCAP 1608 (2016) 036, [1606.02073].

[14] F. Bazzocchi, M. Lattanzi, S. Riemer-Sørensen and J. W. F. Valle, X-ray photons from late-decaying majoron dark matter, JCAP 0808 (2008) 013, [0805.2372].

[15] J. Heeck, Lepton flavor violation with light vector bosons, Phys. Lett. B758 (2016) 101-105, [1602.03810].

[16] BELLE collaboration, T. Yoshinobu and K. Hayasaka, MC study for the lepton flavor violating tau decay into a lepton and an undetectable particle, Nucl. Part. Phys. Proc. 287-288 (2017) 218-220.

[17] J. Heeck and W. Rodejohann, Lepton Flavor Violation with Displaced Vertices, 1710.02062. 